\title{
Study of the functional activity of mucous membrane cervix and vagina in patients with genital herpes
}

\begin{abstract}
In the structure of diseases that affect women reproductive health, viral infection has a special role. There were examined 66 patients with recurrent genital herpes. The data on significant disorders of the lower genital organs mucous membranes, such as functional cell activity, immunological resistance and microbiocenosis in women with recurrent genital herpes is given.
\end{abstract}

Keywords: herpetic infection, diagnosis, clinic, immunological, resistance, recurrent, microbiocenosis
Volume 8 Issue 2 - 2019

Benyuk V, Shcherba E, Lastovetska L, Shako V
Department of Obstetrics and Gynecology, University of

Bogomolets National Medical, Ukraine

Correspondence: Shcherba E, Ph. D., Assistant, Department of Obstetrics and Gynecology, University of Bogomolets National Medical, Kyiv, Ukraine, Email sherbaelena@ukr.net

Received: February 16, 2018 | Published: April 03, 2019

\section{Introduction}

The incidence of viral urogenital infections continues to increase rapidly, being not only a medical, but also a social and economic problem. According to modern research, more than $90 \%$ of people are infected with the herpes simplex virus (HSV). According to official data, 15 cases of genital herpes per 100,000 populations are recorded in Ukraine, in Russia this indicator reached 31\%, however, real statistics are much higher. In European countries, $10-40 \%$ of pregnant women are seropositive for herpes simplex virus type 2 (HSV-2), causes the development of genital herpes $(\mathrm{GH}) .{ }^{1}$ There are 6 types of herpes simplex virus (HSV). GH is caused by the two most common serotypes-HSV-1 and HSV-2, which in accordance cause herpes skin and genital herpes. However, recently there have been reports that HSV of different types can turn from one into another. ${ }^{2}$ With neurodermatropism, HSV affects the skin and mucous membranes (most often on the face and in the genital area), the central nervous system (meningitis, encephalitis), eyes (conjunctivitis, keratitis). HSV causes the pathology of pregnancy and childbirth, which often leads to spontaneous abortion and intrauterine fetal death or causes a generalized infection in newborns. The connection of $\mathrm{GH}$ with cervical cancer and prostate cancers is noted. The GH is transmitted mainly through sexual contact or acquired by the child in childbirth from a sick mother during passage through the birth canal. ${ }^{3}$

Gateway infection is the skin and mucous membranes. The virus gets on the mucous or damaged skin with saliva, sperm or as a result of contact with herpetic lesions of the skin and mucous membranes of a sick person. After penetrating the HSV, it remains in the human body for life in the form of a latent infection. ${ }^{4}$ However, the state of commensalism is not permanent. Herpetic infection can become more active, passing into clinically pronounced forms under the influence of laxative factors (Figure 1). ${ }^{4}$ The risk factors for the onset of herpetic infection are: a large number of sexual partners, an early onset of sexual activity, and a low social level. Factors contributing to the manifestation and/or relapse of GH include: decreased immunological reactivity, fatigue, menstruation, hypothermia and overheating of the body, concomitant infections, medical manipulations, including abortion and the introduction of an intrauterine device, some mental and physiological conditions. ${ }^{1}$

HSV causes lesions of the reproductive organs. Clinical manifestations of genital herpes in women are more pronounced than in men. Symptoms of the disease appear in 2-14 days after contact. First, we notice common symptoms (fever, muscle, joint and headaches). A few days later, local symptoms appear - characteristic painful, bubble elements, merge into clusters. They quickly open, revealing an erosive surface. Herpetic infections are often accompanied by the attachment of a secondary bacterial flora. ${ }^{5}$ Quite often GG has an erased form, or it proceeds atypically. Classical symptoms of GG are observed only in $20 \%$ of patients. A significant proportion of patients have minimal clinical manifestations, which can be ignored. Recurrence of infection usually occurs in people who have a specific combination of immunodeficiency. ${ }^{6}$ Given the high incidence of women, infectious nature, recurrent course, immunity, the leading role in determining the nature of the course of herpetic infection belongs to the state of the system of anti-infectious resistance (SAIR). ${ }^{7}$

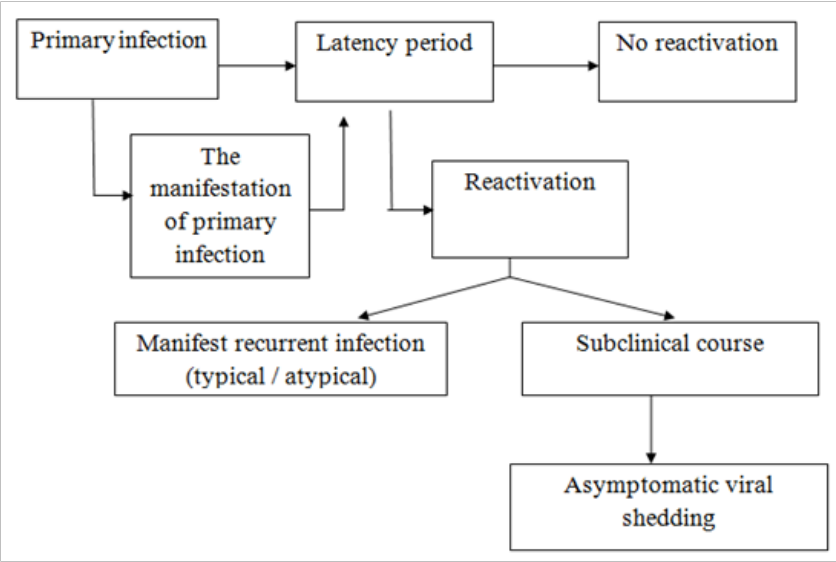

Figure I Course of herpetic infection.

Now the situation has changed qualitatively; new methods of diagnosis of herpes infection based on molecular biological principles have developed effective treatments. Effective methods of treatment are being developed. Doctors formed an understanding of the importance of the problem of $\mathrm{GH}$ and its complications, primarily related to human reproductive health and neonatal pathologies. ${ }^{8}$ The introduction of modern innovative technologies to diagnose the inflammatory process at the cellular level makes it possible to increase the sensitivity and accuracy of diagnostic manipulations and therapeutic and rehabilitation measures. 
Today, the study of the metabolic state of the mucous membranes continues not only on the organ, but also on the cellular level. In our opinion, a promising area is the study of the electrical properties of the cells of the mucous membranes of the genital organs. As a measure of the electro kineticity of the cell nucleus, the percentage of cell nuclei is used to carry a negative electric charge. ${ }^{2}$ The current study aimed to study the effect of genital herpes on the cervix and vagina mucous membranes by observing their functional activity in fertileaged women.

\section{Materials and methods}

We examined 86 women aged 18 to 30 years. The main group consisted of 66 women with recurrent genital herpes. All patients were seropositive for herpes simplex virus type 2 . The control group included 20 healthy women. All subjects had common age and data of obstetric-gynecological and somatic anamnesis. Verification of the diagnosis of recurrent GG was conducted on the basis of anamnesis, epidemiological, clinical and laboratory studies using general clinical blood and urine bacterioscopic studies of vaginal discharge and cervical canal, ELISA of serum for detection of antibodies and PCR of serum and cervical mucus to determine the viral DNA to HSV-2. Also in serum and cervical mucus wedetermined the level of IgM, IgG, IgA sIgA by sandwich ELISA method. In order to determine the effect of clinical signs of disease on the function of cells of the mucous membranes of the vagina and cervix we studied the properties of electro kinetic methods to VI Shakhbazov as modified V.A. Benyuk and O.A. Scherba. Statistical analysis of the results of research conducted on the Pentium-166 using "Exel 2003"program and statistical software "Statistica for WINDOWS v.4.3". The differences are considered significant at $\mathrm{p}<0.05$.

\section{Results}

When identifying the causative agents of the herpes virus (PCR, ELISA), the genital type was determined in all examenees. Analyzing the age structure we found that the risk group is the category of those aged 20 to 25 years $(34,9 \%)$. By studying the medical history, we found that in $93,3 \%$ of women surveyed obstetric and gynecological history were burdened. Due to the analysis of the structure of the transferred gynecological diseases we found that the largest group consisted of patients with STIs - 37 women $(56,0 \%)$. Spontaneous abortion was observed in 35,5\% of women, missed abortion-in $12,2 \%$ abortions in later stages (16-21 weeks) $-8,8 \%$ premature births $-4,4 \%$ inspected. In $20 \%$ of patients diagnosed with menstrual dysfunction in $17,7 \%$-infertility. Dyspareunia was observed in $47,7 \%$ of cases. In all 66 women surveyed were identified with clinical manifestations of HS. Prodormal period averaged 2.3 days and was accompanied by a $50 \%$ morbidity in $80 \%$ of heartburn in $34,4 \%$ - itching in the area of the vulva and vagina in $13,3 \%$-dysuria phenomenon. During the initial evaluation we found that $61(92,4 \%)$ patients were observed with prolonged and heavy vaginal discharge. Herpetic elements have been found on the mucous membrane of the vulva, vagina, small and large labia, perineum. In the background defined erythematous vesicles as small as $2 \mathrm{~mm}$, the disclosure of which was formed after 3-5 days erosions and ulcers. With the progression was observed during epithelization of slow or no crust. Full of disappearance occurred 8-10 days. In $98,1 \%$ was expressed pain, itching, heartburn. All women suffered from 3 to 8 years $(4,5 \pm 0,6)$. The frequency of relapses mild course of herpes infection was observed in $43,3 \%$ of cases (relapse to 2 times a year); average degree - in 34,4\% (recurrence $4-5$ times a year), and $8(12,1 \%)$ patients noted severe degree of disease exacerbations every month. Most relapse took place on 3-4 years of disease (Figure $2)$. Recurrence triggers of the disease were: transitional weather conditions $(45,3 \%)$, stress $(13,7 \%)$, menstruation $(18,5 \%)$, disordered sex life $(22,5 \%){ }^{9}$

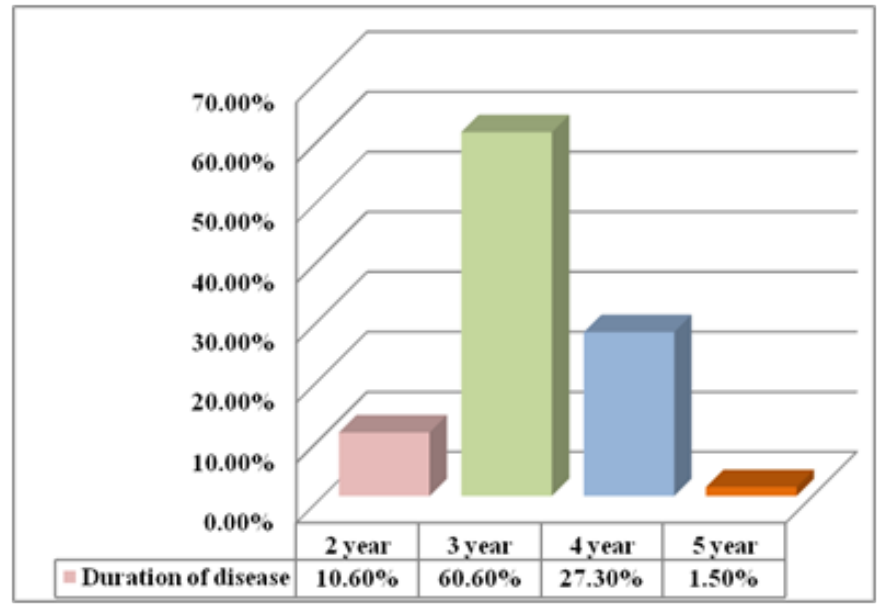

Figure 2 Disease duration of the surveyed women, $n=66$ (\%).

When colposcopy study, in addition to edema and hyperemia of the mucosa, which is typical for inflammation of the cervix and vagina, we diagnosed ectopic prismatic epithelium in $20(30,3 \%)$ cases, atypical transformation zone $10,6 \%$ of leukoplakia $6 \%$, acquired cyst transformation zone in $12,1 \%$ Iodnegativezone in $15,3 \%$ of patients withGG (Figure 3). ${ }^{10}$ In a bacterioscopic examination, the majority of patients had a third degree of purity of the vaginal secretion (Figure 4).

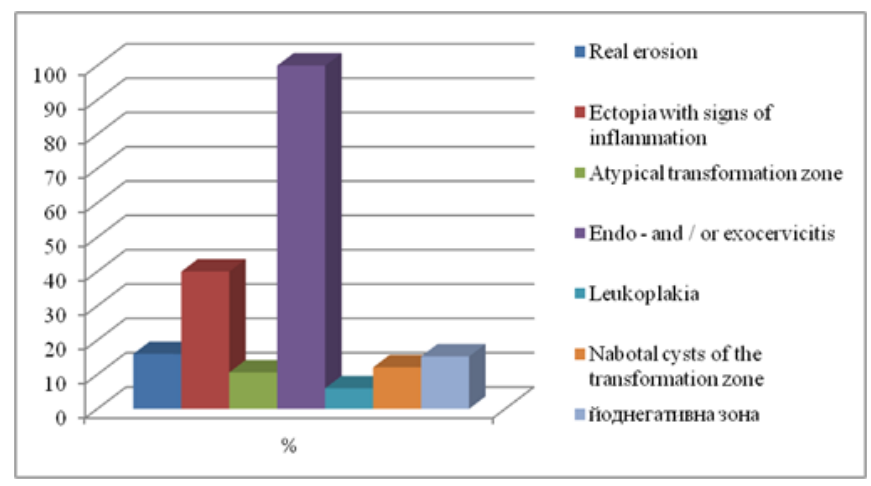

Figure 3 Colposcopic study results of patients with GG, n = 66 (\%).

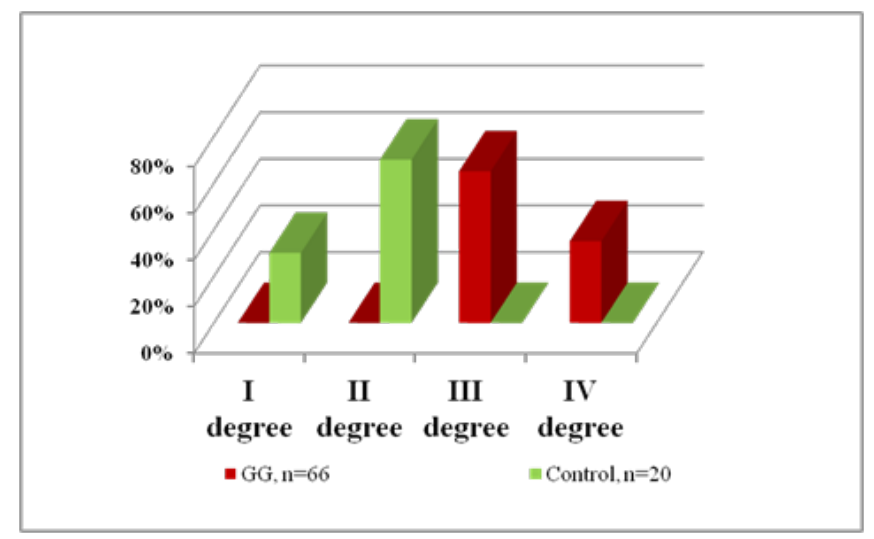

Figure 4 Degree of the vaginal secret purity of the examined women, $n=$ $66(\%)$. 
During the bacteriological study of patients, we found that the majority of the examined patients with GG had a diagnosis of the condition of vaginal dysbiosis, in which there was a sharp decrease or total absence of lactobacilli, abundant polymorphic gram-positive and gram-negative rod and coccal flora. Among the opportunistic flora peptostreptococci $(43,3 \%)$, corynebacteria vaginalis $(57,6 \%)$ and staphylococcus epidermal $(66,7 \%)$ predominated (Figure 5). Thus, changes in the biotope of the vagina, clinical manifestations of GG in patients of reproductive age demonstrate disturbances in the state of the microbiocenosis of the vagina.The analysis of the obtained data revealed significant violations on the part of local immunity in all patients with exacerbation of chronic recurrent GG. The content of secretory IgA and lysozyme in cervical mucus have significantly decreased in almost 5 times in comparison with the corresponding index of the control group $(\mathrm{p}<0.05)$ (Table 1$)$.

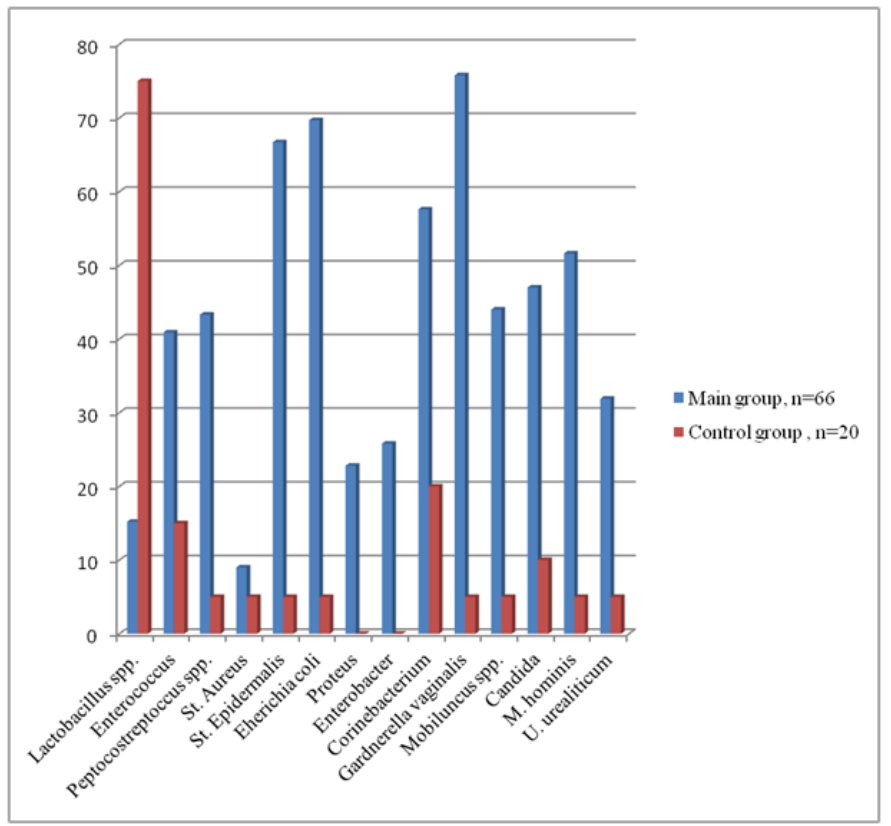

Figure $\mathbf{5}$ Indices of vaginal microbiocenosis in women of reproductive age with recurrent genital herpes.

Table I Indicators of humoral immunity in cervical mucus of women with recurrent genital herpes, $g / l$

\begin{tabular}{lll}
\hline Indicators & \multicolumn{2}{l}{ Group research } \\
\hline & Main group & Control group \\
\hline $\lg M$ & $2,7 \pm 1,08^{*}$ & 0 \\
$\lg G$ & $16,8 \pm 1,45^{*}$ & $0,41 \pm 0,09$ \\
$\lg A$ & $0,18 \pm 0,43$ & $0,13 \pm 0,02$ \\
$\operatorname{sig} A$ & $0,35 \pm 0,08^{*}$ & $1,75 \pm 0,12$ \\
Lysozyme & $0,07 \pm 0,04^{*}$ & $0,33 \pm 1,1$ \\
\hline
\end{tabular}

When studying the electro kinetic activity of squamous epithelial cells in women with recurrent GG, we found a decrease in the number of mobile cells by a factor of 2, a decrease in the index of mobile / immobile cells by 11,4 times; A decrease in average speed of cell nucleusrun by 7,5times, a decrease in the displacement amplitude of the nuclei by 1,6times, a decrease in the amplitude of the displacement of plasmin by 2times, a decrease in the ratio of the displacement amplitude of plasmin to a shift of the nuclei 1,4 times with respect to the control group, A decrease in the intensity of metabolism in the cells of the mucous membrane of the vagina and cervix in the conditions of GG.

We established a correlation between the clinical picture of the disease, local immunity status factors, and the electro kinetic activity of the cells of the flat epithelium of the vagina and cervix.

\section{Conclusion}

a) Changes in the superficial epithelium of the cervix with exacerbation of genital herpes is accompanied by a decrease in protective functions, creates conditions for the rapid development of violations of the biocenosis of the vagina. As a result, the sensitivity to any damage increases; it can cause the development of both background and precancerous processes of the cervix.

b) With infectious diseases of the cervix and vagina, especially caused by virus infections such as genital herpes, it is advisable to investigate the electro kinetic properties of cells of the flat epithelium, it can serve as a criterion of severity and a marker for the course of the inflammatory process.

\section{Acknowledgments}

None.

\section{Conflicts of interest}

Authors declare that there is no conflict of interest.

\section{References}

1. Benyuk VA. Features of complex interferon therapy for recurrent genital herpes. Women's Health. 2013;5(81):25-30.

2. Kovalchuk $\mathrm{Kh} \mathrm{V}, \mathrm{Bu}$ Weiwei, Neimark AS, et al. A modern view of the diagnosis of the state of vaginal cells in women with genital herpes from the perspective of nanotechnology. Ukrainian Scientific Medical Journal of Youth, Special Issue. 2015;2(87):127-128.

3. Krasnopolsky VI, Serova OF, Tumanova VA. Influence of infections on the reproductive system of women. Russian bulletin of the obstetriciangynecologist. 2004;5:26-29.

4. Kuzmin VN. Modern view of the kinetic course, diagnosis and therapy of genital herpes in women. MMA Media Medica. 2014;16(6):55-60.

5. Nitephor IB. State of cellular immunity in women with viral urogenital infection and spontaneous miscarriages. Bulletin of Scientific Research. Ternopil. 2005;2(3):140-141.

6. Osipova LS. Genital herpes. Features of the current and treatment in modern conditions. Ukrainian Journal of Dermatology, Venereology, Cosmetology. 2012.10 p.

7. Panchenko LA. Herpes. Role in human pathology. Antiviral drugs. Kharkov: "Planet-Print". 2014. 204 p.

8. Benyuk V. Modern aspects of correction of vaginal microbiocenosis violation in women with genital herpes. Wschodnioeropejskie Czasopismo Naukowe. 2015;4:156-160.

9. Johnston C. Current concepts for genital herpes simplex virus infection: diagnostics and pathogenesis of genital tract shedding. Clin. Microbiol. Rev. 2016;29(2):149-161.

10. Le Goff J. Diagnosis of genital herpes simplex virus infection in the clinical laboratory. Virol. J. 2014:11:25-83. 